
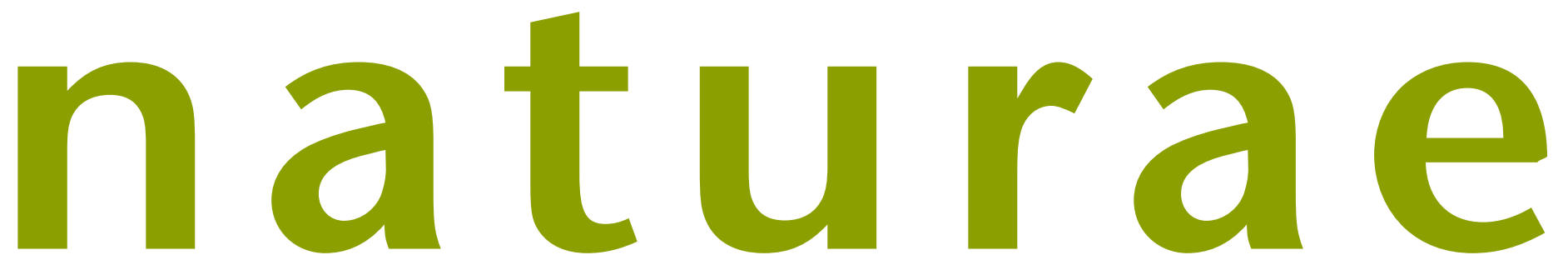

$2021 \cdot 4$

COLLOQUE NATIONAL DE MALACOLOGIE CONTINENTALE, NANTES, 6 ET 7 DÉCEMBRE 2018

Édité par Lilian LÉONARD

Vertigo moulinsiana (Dupuy, 1849) et Vertigo angustior Jeffreys, 1830 en Sarthe: bilan de deux années d'étude

Rémi GERBER, Franck NOËL \& Romain MARIOT 
DiRECTEUR DE LA PUBLICATION / PUBLICATION DIRECTOR: Bruno David,

Président du Muséum national d'Histoire naturelle

RÉDACTEUR EN CHEF / EDITOR-IN-CHIEF: Jean-Philippe Siblet

ASSISTANTE DE RÉDACTION / ASSISTANT EDITOR: Sarah Figuet (naturae@mnhn.fr)

Mise EN PAGE / PAGE LAYOUT: Sarah Figuet

COMITÉ SCIENTIFIQUE / SCIENTIFIC BOARD:

Luc Abbadie (UPMC, Paris)

Luc Barbier (Parc naturel régional des caps et marais d'Opale, Colembert)

Aurélien Besnard (CEFE, Montpellier)

Vincent Boullet (Expert indépendant flore/végétation, Frugières-le-Pin)

Hervé Brustel (École d'ingénieurs de Purpan, Toulouse)

Patrick De Wever (MNHN, Paris)

Thierry Dutoit (UMR CNRS IMBE, Avignon)

Éric Feunteun (MNHN, Dinard)

Romain Garrouste (MNHN, Paris)

Grégoire Gautier (DRAAF Occitanie, Toulouse)

Olivier Gilg (Réserves naturelles de France, Dijon)

Frédéric Gosselin (Irstea, Nogent-sur-Vernisson)

Patrick Haffner (UMS PatriNat, Paris)

Frédéric Hendoux (MNHN, Paris)

Xavier Houard (OPIE, Guyancourt)

Isabelle Le Viol (MNHN, Concarneau)

Francis Meunier (Conservatoire d'espaces naturels - Hauts-de-France, Amiens)

Serge Muller (MNHN, Paris)

Francis Olivereau (DREAL Centre, Orléans)

Laurent Poncet (UMS PatriNat, Paris)

Nicolas Poulet (OFB, Vincennes)

Jean-Philippe Siblet (UMS PatriNat, Paris)

Laurent Tillon (ONF, Paris)

Julien Touroult (UMS PatriNat, Paris)

COUVERTURE / COVER:

Vertigo moulinsiana (Dupuy, 1849). Crédit photo: R. Gerber.

\section{MUSÉuM * Nantes

Naturae est une revue en flux continu publiée par les Publications scientifiques du Muséum, Paris Naturae is a fast track journal published by the Museum Science Press, Paris

Les Publications scientifiques du Muséum publient aussi / The Museum Science Press also publish:

Adansonia, Zoosystema, Anthropozoologica, European Journal of Taxonomy, Geodiversitas, Cryptogamie sous-sections Algologie, Bryologie, Mycologie, Comptes Rendus Palevol.

Diffusion - Publications scientifiques Muséum national d'Histoire naturelle

CP 41 - 57 rue Cuvier F-75231 Paris cedex 05 (France)

Tél. : 33 (0)1 40794805 / Fax: 33 (0)1 40793840

diff.pub@mnhn.fr / http://sciencepress.mnhn.fr

(C) Publications scientifiques du Muséum national d'Histoire naturelle, Paris, 2021

ISSN (électronique / electronic): 1638-9387 


\title{
Vertigo moulinsiana (Dupuy, 1849) et Vertigo angustior Jeffreys, 1830 en Sarthe: bilan de deux années d'étude
}

\author{
Rémi GERBER \\ 14 bis Boulevard de Metz, F-35700 Rennes (France) \\ remi.gerber@edu.mnhn.fr \\ Franck NOËL \\ La Motte, F-53160 Saint-Martin-de-Connée (France) \\ noelfranck@yahoo.fr \\ Romain MARIOT \\ CPIE Vallées de la Sarthe et du Loir, \\ La Bruère, F-72200 La Flèche (France) \\ rmariot@cpie72.fr
}

Soumis le 8 avril 2019 | Accepté le 10 juin 2020 | Publié le 9 février 2021

MOTS CLÉS

Vertigo moulinsiana Vertigo angustior milieux humides, écologie, répartition
Gerber R., Noël F. \& Mariot R. 2021. - Vertigo moulinsiana (Dupuy, 1849) et Vertigo angustior Jeffreys, 1830 en Sarthe: bilan de deux années d'étude, in Léonard L. (éd.), Colloque national de malacologie continentale, Nantes, 6 et 7 décembre 2018. Naturae 2021 (4): 49-55. https://doi.org/10.5852/naturae2021a4

\section{RÉSUMÉ}

Une étude de trois ans, de 2017 à 2019, a été menée en Sarthe par le Centre permanent d'Initiatives pour l'Environnement (CPIE) Vallées de la Sarthe et du Loir sur la famille des Vertiginidae (Gastropoda: Pulmonata) et plus spécifiquement sur Vertigo moulinsiana (Dupuy, 1849) et Vertigo angustior Jeffreys, 1830. Cette étude a pour objectifs d'améliorer la connaissance de leur répartition et de leur écologie et de sensibiliser les acteurs locaux: naturalistes et gestionnaires. Nous présentons ici les principaux résultats des deux premières années d'études consacrées à l'inventaire de Vertigo moulinsiana et Vertigo angustior. La sélection des sites à inventorier est réalisée en deux étapes. D’abord le choix d'une zone d'étude, bassin versant ou site Natura 2000, puis celui des stations à prospecter à partir des cartographies existantes ou par photo-interprétation. Ce sont finalement 524 stations qui ont été prospectées sur deux ans. Quarante-neuf stations abritent V. moulinsiana, sept Vertigo angustior et neuf contiennent les deux espèces. Leur répartition dans le département de la Sarthe est très hétérogène. La vallée des Cartes abrite la grande majorité des stations à Vertigo angustior et de nombreuses localités de Vertigo moulinsiana, les deux espèces pouvant être répertoriées au sein du même site. Vertigo moulinsiana est retrouvé ponctuellement sur d'autres bassins versants comme ceux de la Fare et du Tripoulin.

\section{ABSTRACT}

Vertigo moulinsiana (Dupuy, 1849) and Vertigo angustior Jeffreys, 1830 in Sarthe: two years study assessment.

A three-year study, from 2017 to 2019, was conducted in Sarthe by the CPIE Vallées de la Sarthe et du Loir on the family Vertiginidae (Gastropoda: Pulmonata) and specifically on Vertigo moulinsiana (Dupuy, 1849) and Vertigo angustior Jeffreys, 1830. The aims of this study are to improve knowledge of their distribution and ecology and to raise awareness among local actors as naturalists and managers. The selection of sites to be prospected is carried out in two stages. First, the choice of a study area, catchment basin or Natura 2000 site. Then the choice of stations to be prospected, based on existing maps or by photo-interpretation. 
KEY WORDS

Vertigo moulinsiana

Vertigo angustior

wetlands,

ecology,

distribution.
In the end, 524 stations were prospected in two years. Forty-nine stations contain $V$. moulinsiana, seven for $V$. angustior and nine contain both species. Their distribution is very heterogeneous across the department. Les Cartes valley welcome the vast majority of the $V$. angustior population and many of the $V$. moulinsiana, both species can be found within the same site. $V$. moulinsiana is occasionally found in other catchment basins such as those of the Fare and Tripoulin.

\section{INTRODUCTION}

Vertigo angustior Jeffreys, 1830, le Vertigo étroit et Vertigo moulinsiana (Dupuy, 1849), le Vertigo de Des Moulins, sont deux minuscules gastéropodes terrestres inscrits à l'annexe II de la directive 92/43/CEE du Conseil du 21 mai 1992 concernant la conservation des habitats naturels ainsi que de la faune et de la flore sauvages (directive Habitats-Faune-Flore [DHFF]). Ces deux espèces sont largement répandues à l'échelle du territoire métropolitain, essentiellement sur socle calcaire, mais leurs populations restent encore mal connues à l'échelle locale et de nombreuses lacunes subsistent dans la cartographie de leur aire de distribution (Muséum national d'Histoire naturelle Inventaire national du Patrimoine naturel [INPN] : https://inpn. mnhn.fr, dernière consultation le 3 mai 2020 )

En Sarthe, les auteurs du début du siècle ne connaissaient aucune de ces deux espèces, qui d'ailleurs ne sont pas citées dans le recueil des Mollusques du Maine de l'Abbé Letacq (1924), couvrant plus largement le Maine et la Basse-Normandie. $V$. moulinsiana est signalé pour la première fois en 2007 (Hesnard \& Noël 2012) et $V$. angustior plus récemment encore (obs. pers.), à la faveur d'une étude visant la Bythinelle de la Sarthe Bythinella turriculata (Paladilhe, 1869). Il est cependant possible qu'une mention ancienne citée d'Avessé «au bord des sources des prés de la Guyonnière» sous le nom de V. pusilla (Letacq 1924) se rapporte à $V$. angustior du fait de son écologie. Toutefois, cette station n'a pas pu être retrouvée et confirmée. Ces récentes découvertes, associées au statut des deux espèces, à l'absence de la prise en compte des Mollusques dans les documents d'objectifs Natura 2000 (DOCOB) en Sarthe et à une forte potentialité d'habitats favorables, ont permis la mise en place d'un projet d'étude de trois ans, de 2017 à 2019, sur les Vertiginidae. Ce projet est porté par le CPIE Vallées de la Sarthe et du Loir, financé par la Région Pays de la Loire et soutenu par l'Agence de l'Eau. Trois axes sont développés:

- connaître: répartition des espèces et facteurs écologiques expliquant cette répartition;

- faire connaitre: sensibilisation des acteurs locaux, naturalistes et gestionnaires d'espaces naturels;

- gérer et protéger: expérimentation d'axes de gestion.

\section{MATÉRIEL ET MÉTHODES}

\section{SÉLECTION DES SITES À PROSPECTER}

L'établissement d'une cartographie d'occurrence des deux Vertigos a nécessité plusieurs étapes. Étant donné l'inscription des deux Vertigos à l'annexe II de la DHFF et leurs affinités pour les milieux humides (Cameron et al. 2003), plusieurs bassins versants et sites Natura 2000 a priori favorables à la présence d'au moins une des deux espèces ont été sélectionnés (Fig. 1). La sélection des sites à prospecter a été établie à partir de la connaissance du terrain des porteurs de ce projet, et du croisement de la couche de pré-localisation des zones humides probables en Sarthe (Geo Data Gouv: https://geo.data.gouv. $\mathrm{fr} / \mathrm{fr} /$, dernière consultation le 3 mai 2020) avec les couches pédologiques (IGN [s. d.], Geoportail: www.geoportail.gouv. fr, dernière consultation le 3 mai 2020). L'objectifétait de délimiter de grandes zones du territoire qui comprendraient des zones humides calcaires à neutres, favorables aux $V$. angustior et $V$. moulinsiana. Nous nous sommes concentrés la première année sur les bassins versants de l'Orne Saosnoise, de la Vègre, du Dué et du Narais et sur le site Natura 2000 FR5200647 «Vallée du Narais, forêt de Bercé et ruisseau du Dinan ». La deuxième année a permis de prospecter les bassins versants de la Veuve, de l'Aune, de la Fare et de la Marconne et le site Natura 2000 FR5200649 «Vallée du Loir de Vaas à Bazouge».

Le choix des stations à prospecter s'est effectué de deux façons différentes:

- pour les sites Natura 2000, l'existence d'une cartographie des habitats a permis la sélection des zones humides à partir des critères de végétation: cariçaie, roselière, bas marais, etc. (Drake 1999; Killeen 2003; Cameron et al. 2003; Książkiewicz-Parulska \& Ablett 2016);

- au sein de ces bassins versants, les stations ont été présélectionnées par photo-interprétation (Zones Humides. 15 septembre 2015: http://www.zones-humides.org/, dernière consultation le 3 mai 2020). Sur les photos-aériennes, les zones humides sont en général plus vertes que les zones non humides et les cariçaies et mégaphorbiaies se distinguent des autres habitats par une hétérogénéité des milieux avec notamment des touradons de Carex (Fig. 2). Sur le terrain, seules les zones effectivement humides, c'est-à-dire avec une litière humide, ont été prospectées.

En complément, des sites découverts lors du passage d'une parcelle à une autre, qui apparaissaient favorables mais qui n’ont pas été sélectionnés car trop petits ou cachés par des arbres lors de la pré-sélection, ont également été visités et ajoutés à la liste.

\section{PérIOde D'INVENTAIRE}

L'inventaire a eu lieu tout au long des deux années d'études, en évitant les jours de pluie. Entre avril et juin, les prospections ont été plus importantes en raison de la présence des stagiaires accueillis par le Centre permanent d'Initiatives pour l'Environnement (CPIE). 


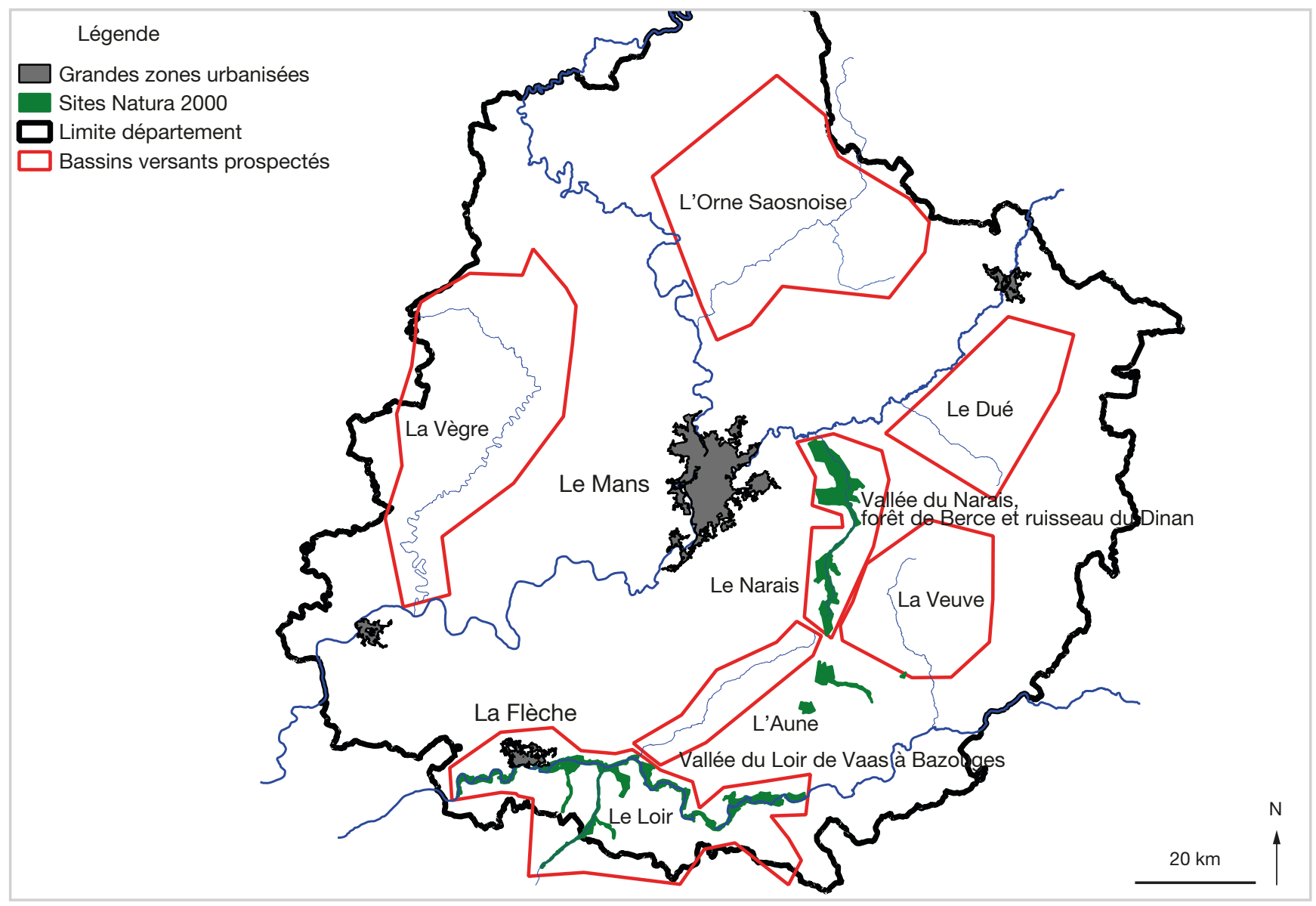

FIG. 1. - Zones de prospection de Vertigo moulinsiana (Dupuy, 1849) et Vertigo angustior (Jeffreys, 1830) en Sarthe. Source: R. Gerber, CPIE Vallées de la Sarthe et du Loir, IGN.

TECHNiques DE DÉTECTION

En 2017, trois techniques de détection ont été appliquées dans chaque station suivant Cucherat \& Demuynck (2008): une recherche à vue pendant 15 minutes par station, un battage de végétation dans un quadrat de $50 \times 50 \mathrm{~cm}$ et un prélèvement de litière sur le même quadrat. Pour le battage de végétation, celle-ci est coupée rase puis secouée au-dessus d'une bassine. En 2018, au regard des résultats de 2017, seule la recherche à vue, à la fois sur la végétation et sur la litière, a été utilisée (Lesage 2017). En effet, 19 stations sur 24 (80\%) avec $V$. moulinsiana ont été détectées à vue. La seule station de $V$. angustior découverte en 2017 l'a également été par la méthode de recherche à vue.

\section{RÉSULTATS}

\section{STATIONS PROSPECTÉES}

Depuis le début du projet, 524 stations ont été visitées et 329 ont été prospectées (Fig. 3). Les stations non inventoriées correspondent à des sites déterminés comme a priori favorables lors de la sélection des stations mais dont les habitats se sont avérés ne pas correspondre aux exigences des espèces ciblées. 58 stations inventoriées $(17 \%)$ abritaient au moins l'une des deux

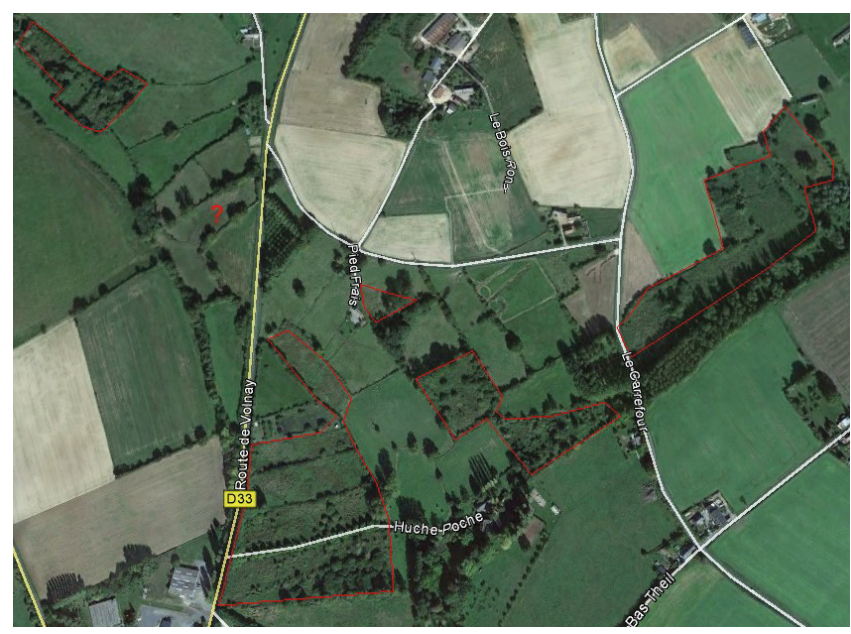

FIG. 2. - Exemple de pré-localisation des habitats à prospecter, encadrée en rouge.

espèces. V. moulinsiana a été retrouvé dans 53 stations, contre seulement 13 stations pour $V$. angustior. Par ailleurs, toutes les stations avec $V$. angustior jouxtent une station à $V$. moulinsiana et neuf stations comptent les deux espèces. La plupart des stations sont proches et se situent au sein d'une même vallée. 


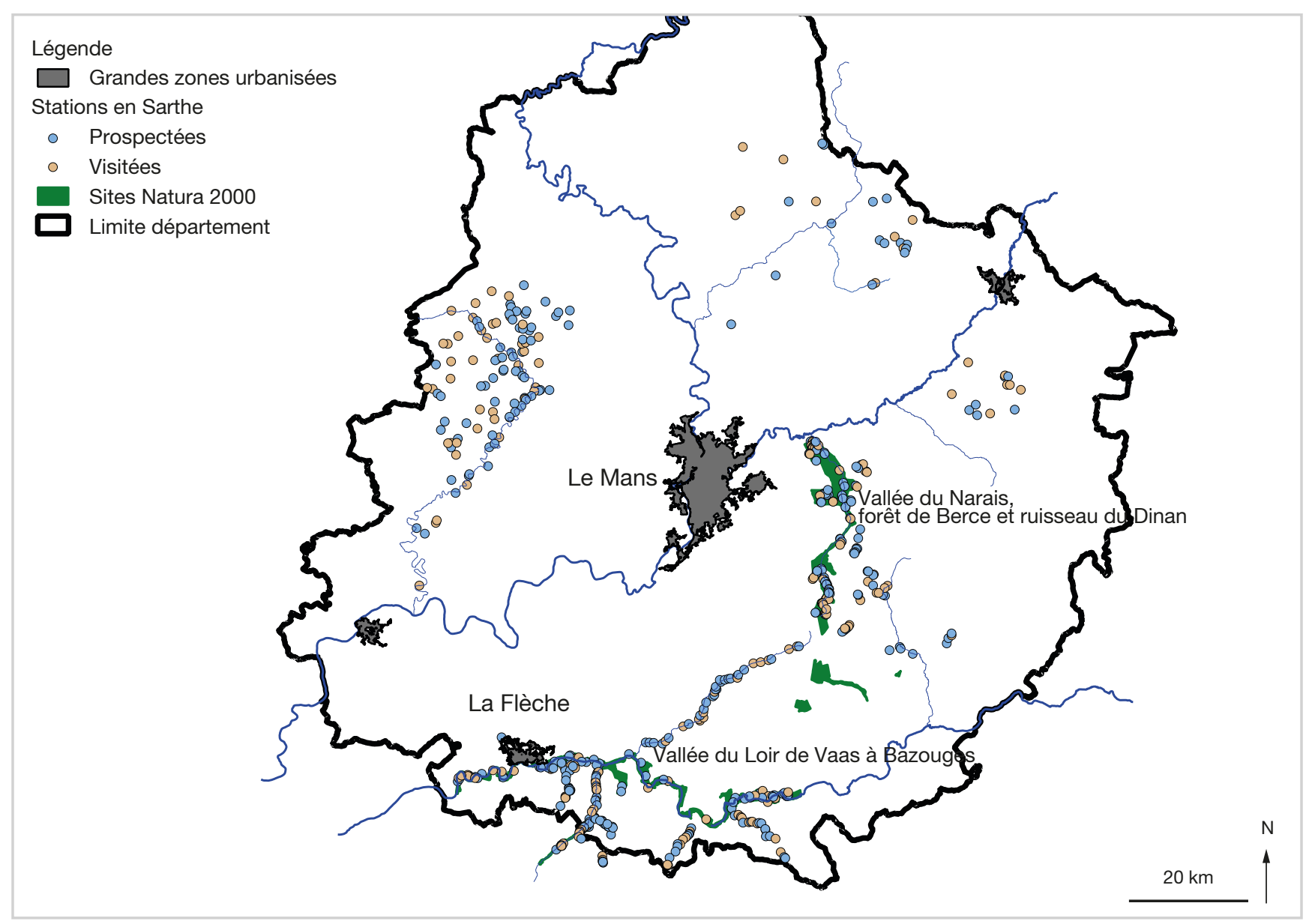

FIG. 3. - Stations visitées et prospectées en Sarthe pour la recherche du Vertigo angustior Jeffreys, 1830 et du Vertigo moulinsiana (Dupuy, 1849). Source: R. Gerber, CPIE Vallées de la Sarthe et du Loir, IGN.

Citons par exemple le Leuray, les Cartes, le Palais, la Longuève, le Narais et ses affluents, ainsi que le nord de la Veuve (Fig. 4).

Des stations ponctuelles ont également été trouvées sur la Fare, l'Aune et le Tripoulin (affluent de l'Orne saosnoise). En revanche, seule une station de Vertigo de Des Moulins a été trouvée le long du Loir, malgré un effort de prospection important. Les trois quarts des stations avec $V$. angustior sont situés sur la rivière des Cartes et l'un de ses affluents.

Enfin, plus de $80 \%$ des stations avec $V$. angustior et près de $70 \%$ des stations avec $V$. moulinsiana se situent dans un site Natura 2000.

\section{HABITATS}

Selon les typologies communément utilisées (EUNIS, Corine Biotope, Natura 2000), $V$. angustior et $V$. moulinsiana ne semblent pas affiliés à un type d'habitat particulier. Néanmoins, les résultats des prospections montrent deux grandes catégories d'habitats préférentiels pour $V$. moulinsiana: la première catégorie comprend des végétations très denses composées d'hélophytes et de Carex sp. (essentiellement Carex riparia Curtis et Carex acutiformis Ehrh.) comme les cariçaies, fossés, roselières et mégaphorbiaies (Fig. 5). Le deuxième grand type d'habitat comprend les bas-marais calcaires et les prairies humides. La végétation est moins dense et souvent moins haute, mais le sol reste humide toute l'année. Nous pouvons noter que les habitats secondaires du Vertigo de Des Moulins, comme les boisements ou les fossés, ainsi que la plupart des prairies humides comprennent des taches de Carex sp. ou d'hélophytes dans lesquelles les $V$. moulinsiana sont très souvent regroupés.

$V$. angustior est retrouvé dans des habitats de type prairial, plus ouverts et à végétation moins élevée, comme des bas marais calcaires, des prairies maigres de fauche et des prairies humides de fauche (Fig. 5). Il a aussi été retrouvé à trois reprises dans les même cariçaies et cladiaies que $V$. moulinsiana.

\section{DISCUSSION}

\section{PRÉPARATION DES INVENTAIRES}

Le choix de la photo-interprétation permet d'optimiser le temps passé sur le terrain même s'il ne s'agit pas d'une méthode absolue en termes de détection d'habitats favorables à $V$. moulinsiana et $V$. angustior. Par exemple, il est possible de confondre une zone humide avec certains champs cultivés car le grain et la couleur de ces habitats sur orthophotographie peuvent se 


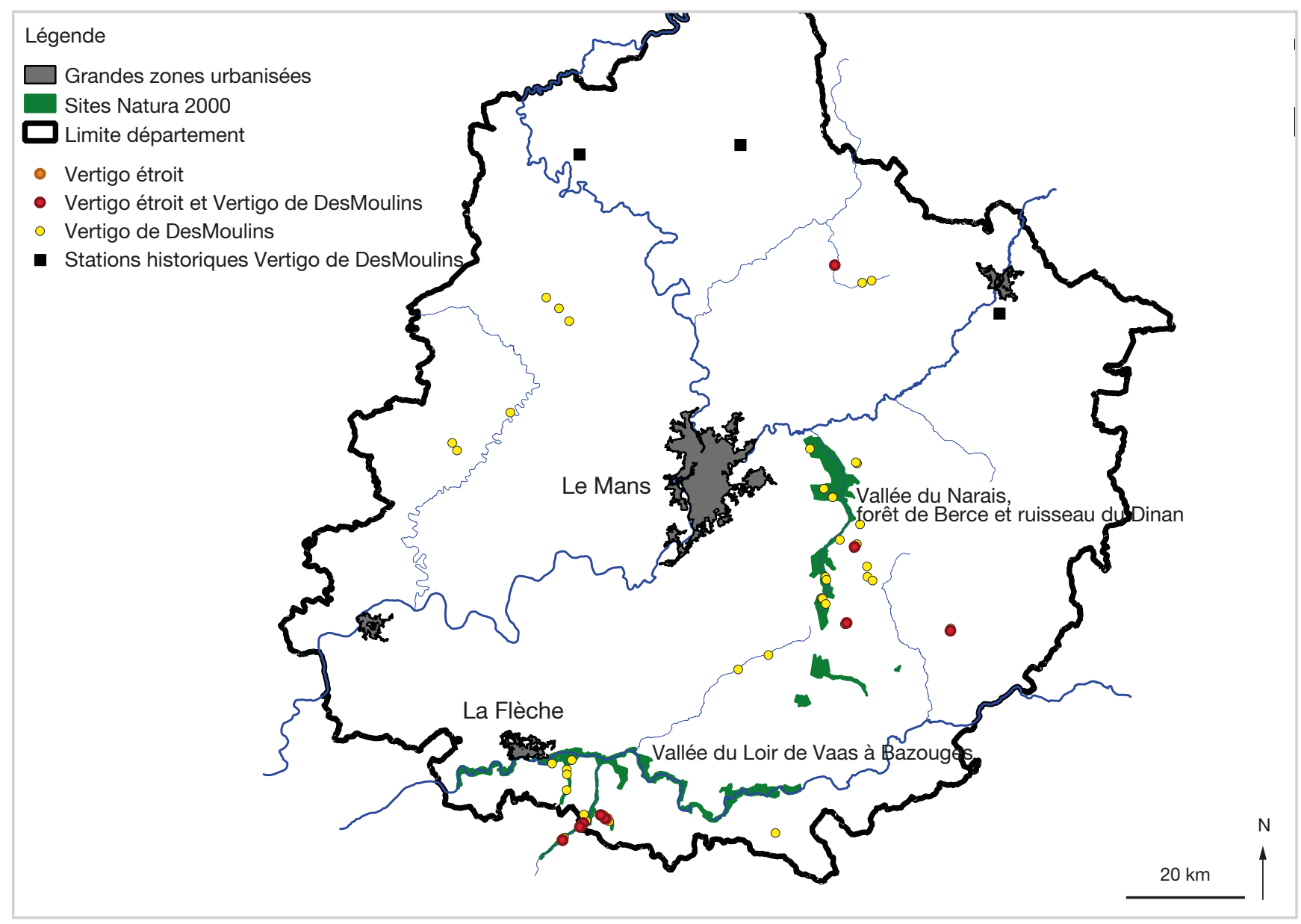

FIG. 4. - Répartition de Vertigo moulinsiana (Dupuy, 1849) et Vertigo angustior (Jeffreys, 1830) en Sarthe. Source: R. Gerber, CPIE Vallées de la Sarthe et du Loir, IGN.

ressembler. De plus, les petites zones humides ou celles sous couvert forestier, comme les peupleraies, échappent à la détection des habitats cibles. En revanche, cette méthode permet in fine d'augmenter l'effort d'échantillonnage en orientant grandement les recherches sur le terrain.

Un des problèmes principaux de la détection des habitats favorables réside dans l'évolution diachronique des paysages et par conséquent des peuplements qui leurs sont inféodés. En effet, certaines roselières ou peupleraies récemment plantées ont peut-être comme origine des prairies à litière dont l'exploitation a été délaissée, ou des pâtures plus ou moins extensives. À l'inverse, certaines prairies marécageuses ont été, par le passé, cultivées et drainées, puis abandonnées du fait de conditions d'exploitation non satisfaisantes (obs. pers.). Faute d'historique disponible pour la plupart de ces milieux en constante évolution (dynamique végétale, gestion), il s'avère difficile de comprendre la répartition et les densités relevées sur chaque site étudié.

\section{EFFICACITÉ DES TECHNIQUES DE DÉTECTION}

La recherche à vue est la technique de détection la plus rentable en termes de temps passé et de capacité de détection. Au sein d'un habitat, la distribution des vertigos est hétérogène (Gerber 2018). Par exemple, il est possible de trouver
$V$. moulinsiana sur une surface d'un mètre carré de Carex et qu'il y soit absent tout autour (obs. pers.). La recherche à vue, associée à l'expérience de recherche des Vertigos, permet de s'orienter rapidement vers ces micro-habitats favorables et d'augmenter l'efficacité de détection de ces espèces. L'effort d'échantillonnage n'est donc pas constant au cours du temps et entre les différents opérateurs, mais il augmente avec l'expérience. Le battage de végétation et le prélèvement de litière prennent plus de temps que la recherche à vue. Même s'il est également possible de s'orienter vers les micro-habitats jugés favorables avec ces techniques, le rapport détection-temps reste inférieur à la recherche à vue.

Le battage de végétation cible principalement $V$. moulinsiana que l'on retrouve régulièrement sur les tiges de plantes comme des Carex sp. Or, cette habitude le rend également facilement détectable à vue. Ceci pourrait expliquer en partie pourquoi le nombre de stations avec $V$. moulinsiana est plus élevé que celui avec $V$. angustior. La technique du prélèvement de litière représente un investissement en temps important et il est difficile d'identifier les endroits les plus adaptés. Cependant, en termes d'abondance relative, le prélèvement de litière permet d'obtenir des densités plus représentatives que les autres méthodes (à vue et battage), avec un nombre plus important de coquilles détectées. Lors de la deuxième 


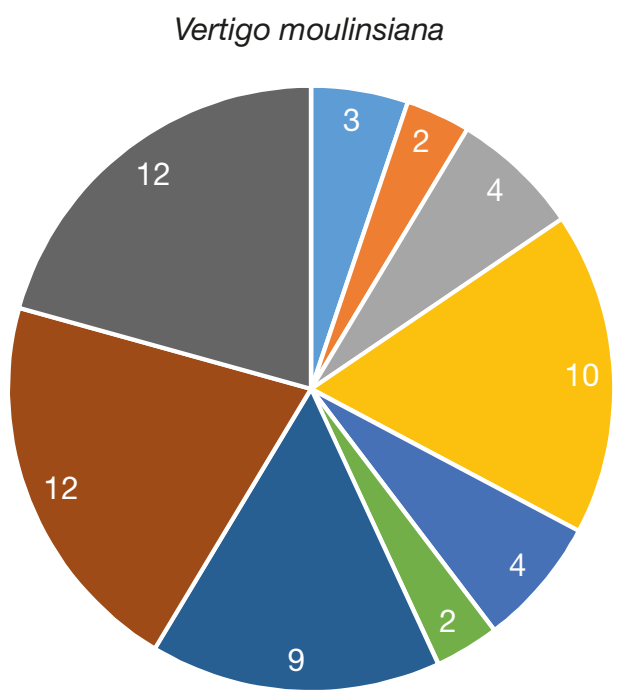

$\square$ Boisement
$\square$ Bord de mare
$\square$ Fossé
$\square$ Mégaphorbiaie
$\square$ Roselière
$\square$ Cladiaie
$\square$ Cariçaie
$\square$ Prairie humide
$\square$ Bas-marais alcalin

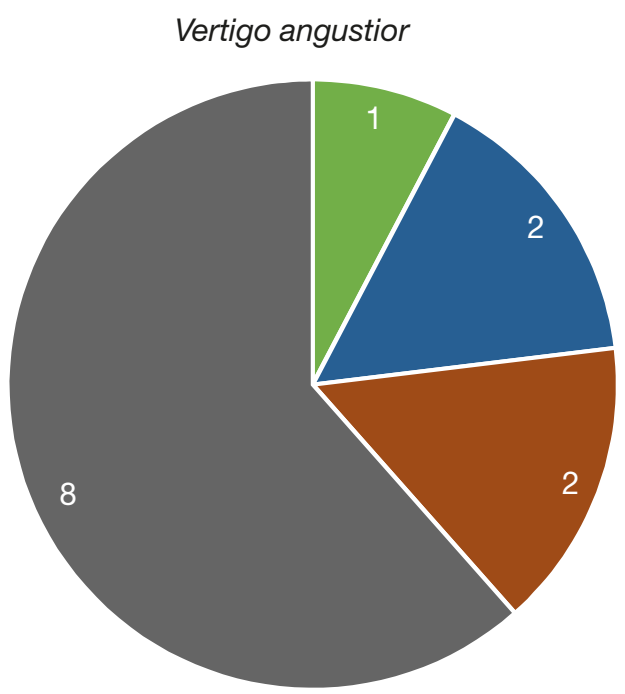

FIG. 5. - Répartition du nombre de stations de Vertigo moulinsiana (Dupuy, 1849) et Vertigo angustior (Jeffreys, 1830) par catégorie d'habitat.

campagne de terrain, en 2018, les prélèvements de litière ont permis de détecter des coquilles de $V$. angustior et de $V$. moulinsiana dans deux stations où aucun individu n'avait été trouvé avec la recherche à vue (Gerber 2018).

Dans cette étude, nous avons privilégié la recherche à vue car elle permet un gain de temps par station inventoriée. Cela nous a permis d'étendre les zones de prospection et par conséquent d'inventorier plus de stations. Nous pouvons également noter que la capacité de détection de $V$. angustior et $V$. moulinsiana augmente rapidement avec la pratique de la recherche à vue. Néanmoins, cette méthode ne permet pas de s'assurer avec certitude de la présence des Vertigos. Il est possible en effet que des populations de faible densité n'aient pas été détectées avec la recherche à vue alors qu'elles auraient pu l'être par prélèvement de litière ou battage. Un compromis a donc été nécessaire: nous avons choisi d'explorer un grand nombre de stations en prenant le risque de manquer celles avec de faibles densités de $V$. moulinsiana ou $V$. angustior.

\section{DISTRIBUTION}

Cette étude a permis la découverte de nombreuses stations avec présence avérée de $V$. moulinsiana et $V$. angustior. En effet quatre stations d'occurrence de $V$. moulinsiana et une seule avec $V$. angustior étaient connues en 2015 dans le département de la Sarthe, contre 62 en 2019 (incluant les stations historiques [Fig. 4] ainsi que celles découvertes lors de notre étude). Ceci est à mettre en relation avec les zones prospectées. Ainsi, parmi ces zones, on constate que les deux vertigos ne sont pas distribués de façon homogène alors que l'effort de prospection et les méthodes de recherche ont été identiques entre les zones prospectées. La distribution des stations semble indiquer la présence de métapopulations plus ou moins continues au sein de grandes zones favorables, comme la vallée des Cartes.
Le long des grandes rivières comme le Loir, les habitats pourtant jugés comme favorables semblent beaucoup moins propices aux vertigos recherchés. Seule une station avec $V$. moulinsiana, au sein d'une roselière à Glyceria maxima (Hartm.) Holmb., a été trouvée. $V$. angustior et $V$. moulinsiana ont besoin d'une hygrométrie constante (Moorkens \& Killen 2011). Les bords du Loir sont inondés en hiver et secs en été, ce qui ne permettrait pas le maintien de populations.

L'état de connaissance actuel et les données récoltées semblent montrer que $V$. angustior est nettement plus rare que $V$. moulinsiana en Sarthe. Ceci est à mettre en perspective avec la meilleure détectabilité de $V$. moulinsiana par la méthode de recherche à vue. En effet, ce dernier est connu pour grimper sur la tige des hélophytes, ce qui le rend plus facile à trouver que $V$. angustior qui tend à rester dans la litière (Cameron et al. 2003). Néanmoins, la recherche à vue a permis de détecter 12 des 13 stations d'occurrence de $V$. angustior. Une autre explication concernant le nombre plus élevé de stations avec $V$. moulinsiana est qu'il trouve plus facilement des zones refuges. En effet, il peut se maintenir dans des zones restreintes tant qu'elles restent constamment humides. Ainsi, il est régulièrement trouvé dans des sites délaissés tels que les fossés à Carex sp. ou roseaux (obs. pers.). Le Vertigo étroit, plus sensible aux inondations de la litière, ne peut pas subsister dans ces milieux régulièrement immergés (Cameron et al. 2003). Cet aspect peut amener à supposer que des zones refuges restreintes où $V$. angustior subsisterait en faible densité n'ont pas été trouvées, cette espèce préférant initialement les écotones entre marais et prairies (Cameron et al. 2003).

L'ensemble des vallées sarthoises n'a pas encore été étudié mais la dispersion des stations et l'isolement de populations au sein de zones à forte dynamique agricole engendre quelques inquiétudes. La fragmentation rend les populations plus sensibles aux aléas climatiques et humains et limite la colonisation d'habitats favorables à l'espèce. Les populations de $V$. angustior connues à ce jour sont pratiquement toutes situées dans le site Natura 2000 de la vallée du Loir, prospecté en 2018, ce qui permettra de prendre en compte l'espèce dans les DOCOB et de mettre 
en place une gestion adaptée des habitats. Néanmoins, plusieurs stations se situent en dehors de sites Natura 2000. Un travail de sensibilisation des propriétaires et gestionnaires est prévu dans la dernière phase du programme.

\section{CONCLUSION}

Notre étude a permis d'augmenter considérablement la connaissance sur la répartition des $V$. moulinsiana et $V$. angustior, répondant ainsi à l'objectif de son premier axe. Pour mieux prendre en compte ces espèces lors de l'élaboration des DOCOB et pour mieux comprendre leurs répartitions, il est important de les faire connaître (deuxième axe de notre étude). Pour cela, deux journées techniques «Vertigo» ont été organisées à Parigné-l’Évêque, la première journée étant consacrée à la sensibilisation des professionnels de l'environnement (gestionnaires de réserves naturelles, techniciens de rivières, bureau d'études, etc.) et la deuxième aux naturalistes et à toutes personnes intéressées par le sujet. Ces journées avaient pour objectifs de faire découvrir les Vertigos et les Mollusques terrestres en général: diversité, écologie, enjeux, etc. Une initiation à l'identification des espèces a également été menée. Cela a permis de réunir différents acteurs locaux de l'environnement. Ainsi, en plus du "porter à connaissance", des échanges avec les gestionnaires de sites permettent d'envisager des pratiques adaptées aux $V$. moulinsiana et $V$. angustior, cela afin d'expérimenter différents axes de gestions possibles.

\section{Remerciements}

Nous voudrions remercier les financeurs de cette étude: la Région Pays de la Loire. Merci également aux relecteurs de l'article, Lilian Léonard et Quentin Wackenheim. Enfin, merci au CPIE Vallées de la Sarthe et du Loir et à toutes les personnes qui ont contribué à ce projet.

\section{RÉFÉRENCES}

Cameron R., Colville B., Falkner G., Holyoak G. A., HorNung E., Killen I. J., Moorkens E., Pokryszko B. M., Von Proschwitz T., TatTersfield P. \& VAlovirta I. 2003. - Species accounts for snails of the genus Vertigo listed in the Annex II of the Habitat Directive: $V$. angustior, $V$. genesii, $V$. geyeri and V. moulinsiana (Gastropoda: Pulmonata: Vertiginidae). Heldia 5 (7): 151-170.

CuCHerAT X. \& DemuYnCK S. 2008. — Les plans d'échantillonnage et les techniques de prélèvements des Mollusques continentaux. MalaCo 5: 244-253.

DraKe C. M. 1999. - A review of the status, distribution and habitat requirements of Vertigo moulinsiana in England. Journal of Conchology 36: 63-79.

GERBER R. 2018. - Amélioration des connaissances de Vertigo moulinsiana (Dupuy, 1849) et Vertigo angustior (Jeffreys, 1830) en Sarthe. Mémoire de Master 2 Expertise Faune Flore. Muséum national d'Histoire naturelle, Paris, $30 \mathrm{p}$.

Hesnard O. \& NoËL F. 2012. — Le Maillot de Des Moulin Vertigo moulinsiana: un mollusque méconnu en Sarthe. Bulletin d'Entomologie tourangelle et ligérienne 33 (2): 44-55

KSIĄŻKIEWICZ-PARULSKA Z. \& ABLETT J. D. 2016. — Investigating the influence of habitat type and weather conditions on the population dynamics of land Snails Vertigo angustior Jeffreys, 1830 and Vertigo moulinsiana (Dupuy, 1849). A Case Study from Western Poland. Journal of Natural History 50 (27-28): 1749-58. https://doi.org/10.1080/00222933.2016.1156178

KiLLEEN I. J. 2003. — Ecology of Desmoulin's Whorl Snail: Vertigo moulinsiana. Ecology of Desmoulins whorl snail Vertigo moulinsiana. Conserving Natura 2000 Rivers. Ecology Series 6: 1-25.

LESAGE B. 2017. - Inventaire de Vertigo moulinsiana et Vertigo angustior sur le département de la Sarthe: dossier de stage. Licence professionnelle Gestion des Ressources environnementales en Milieu rural, Université de Caen-Normandie, Saint Pierre sur Dives, 45 p.

LeTACQ A.-L. (AвBÉ) 1924. - Manuel pour servir à l'étude des Mollusques du Maine et de la Basse-Normandie. Bulletin de Mayenne-Sciences $212 \mathrm{p}$.

Moorkens E. A. \& KilleEn I. J. 2011 - Monitoring and Condition Assessment of Populations of Vertigo geyeri, Vertigo angustior and Vertigo moulinsiana in Ireland. National Parks and Wildlife Service, Department of Arts, Heritage and Gaeltacht (coll. Irish Wildlife Manuals; 55), Dublin, 142 p. 